coronary artery calcium (CAC) score is a surrogate for atherosclerosis that strongly predicts incident coronary artery disease and major CVD events, independent of traditional risk factors. The prevalence of CAC deposition in SLE patients over the age of 45 is known to be significantly higher compared with the Multi-Ethnic Study of Atherosclerosis (MESA) cohort, however data on patients $<45$ years of age is scarce. evaluated CAC scores in younger SLE patients, compared with healthy controls from the Coronary Artery Risk Development in Young Adults (CARDIA) cohort.

Methods We identified 76 SLE patients meeting 1997 ACR classification criteria, without known coronary artery disease and who had a non-contrast CT chest performed as part of their clinical care, with images retrievable for calculation of CAC scores, using the Agatston score. Demographics, disease characteristics, and comorbidities were ascertained. Prevalence of any calcification, defined as CAC $>0$, was reported and compared with data from the CARDIA cohort, a large biracial U.S. cohort of patients ages 33 to 45 at time of chest CT scan for CAC determination. Additionally, within our SLE cohort, we investigated the relationship between disease characteristics and presence of any coronary artery calcification.

Results 76 SLE patients were studied $(40 \pm 13$ years old, 90\% female, 33\% Hispanic, 40\% African American, disease duration $7 \pm 6$ years). Patients met on average $6 \pm 2$ ACR-SLE classification criteria; all had positive ANA titers, $64 \%$ had elevated dsDNA titers. Average SLE disease severity index1 was moder-

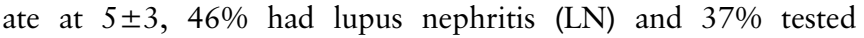
positive for antiphospholipid (APL) antibodies. The prevalence of $\mathrm{CAC}>0$ was $42 \%$ for patients of all ages, $32 \%$ for age $<45,62 \%$ for age 45 . CAC scores were between 1 and 100 in $72 \%$ of the patients and $>100$ for the remaining $28 \%$. When compared with the CARDIA subjects, more SLE patients ages $<45$ had a CAC $>0 \quad(32.0 \%$ vs $9.6 \%$, pvalue $<0.00001)$. Additionally, $29 \%$ of SLE patients age 18 to 32 , with 5 years median SLE disease duration, had abnormal CAC scores; the youngest of whom was 21 years old. SLE patients with CAC were more likely to be older, have a history of a HTN, and have higher BMI. There were no significant differences in SLE disease duration, SLE severity index, APL antibodies, prevalence of $\mathrm{LN}$, or smoking status, between patients with and without CAC.

Conclusions Young SLE patients have a significantly higher CAC scores compared with the general population. A CAC $>0$ was seen in $32 \%$ and $29 \%$ of SLE patients $<45$ and $<33$ years old, respectively. Our data suggest that subclinical atherosclerosis in SLE develops as early as the second decade of life, and warrant screening and cardio-protective interventions.

Funding Source(s): None

\section{INTRACARDIAC TUMOR VS THROMBUS IN PATIENTS WITH ANTIPHOSPHOLIPID SYNDROME: TWO CASE REPORTS}

Consuelo Ramos Giráldez*, Nahia Plaza Aulestia, Maria Luisa Velloso Feijoo, José Luis Marenco de la Fuente. Valme Hospital (Rheumatology Unit)

10.1136/lupus-2019-Ism.145
Background Antiphospholipid syndrome (APS) is characterized by spontaneous and recurrent vascular thromboses, abortion and thrombocytopenia. Cardiac manifestations are rare, but may occur as cardiac masses, such as thrombus (Libman-Sacks endocarditis). On the other hand, it is known that the most common type of primary cardiac tumors, myxoma, can produce clinical pictures similar to APS and SLE, and that tumor exeresis resolves symptoms.

Methods We examined 2 patients diagnosed with SLE and APS that presented cardiac mass. They were assessed with careful history taking, physical examination, laboratory tests, echocardiography and histological examination.

Results Case 1. A 13-years-old girl, with history of autoimmune hemolytic anemia treated with corticosteroids, folic acid and splenectomy a year before, presented a right ischemic stroke with hemiparesis sequel. Laboratory tests revealed ANA, antiDNAds, aCL and lupus anticoagulant positive and echocardiography showed a cardiac mass. She was underwent surgery to exeresis the mass, which was compatible with myxoma. She was diagnosed with immune syndrome secondary to myxoma and did not take treatment. Seven months after the complete exeresis of the myxoma, she was admitted to our hospital because of two months fever, polyarthralgia, oral ulcers and malar rash. Echocardiography showed pericardial effusion and blood tests showed lymphopenia, anemia, ANA and antiDNA positive and elevation of acute phase reactants; urinalysis was normal. She was diagnosed with SLE flare and was successfully treated with corticoids and started hydroxychloroquine, azathioprine and acetylsalicylic acid.

The second case is a 48-years-old woman diagnosed with SLE and associated APS (oral ulcers, thrombosis, arthritis, malar rash, ANA, Coombs and aCL positive), with a history of bilateral iliac arterial ischemia caused by myxoma emboli (confimated by histological examination) and a cardiac mass on echocardiogram. Fourteen months after the vascular surgery, the patient still needs treatment with rituximab, azathioprine and corticoids to control SLE activity and is on anticoagulant treatment to prevent thrombotic episodes.

Conclusions In patients with myxoma and symptoms of APS and/or SLE, there is doubt whether these are secondary to myxoma or if these diseases coexist, so it is recommended to closely monitor clinical activity after exeresis of myxoma and consider not suspending immunosuppressants and/or anticoagulants or the progressive withdrawal of drugs according to symptoms, in order to avoid possible serious complications of autoimmune disease.

Funding Source(s): None

\section{CLINICAL AND IMMUNOLOGICAL RESPONSE OF CHILDHOOD-ONSET SYSTEMIC LUPUS ERYTHEMATOSUS PATIENTS TREATED WITH RITUXIMAB}

${ }^{1}$ Alina Boteanu*, ${ }^{2}$ Maria Angeles Blazquez Cañamero, ${ }^{2}$ Sandra Garrote, ${ }^{2}$ Mariluz Gamir. ${ }^{1}$ Hospital Ramón y Cajal; ${ }^{2}$ University Hospital Ramón y Cajal, Madrid

\subsection{6/lupus-2019-Ism.146}

Background Systemic lupus erythematosus (SLE) is an autoimmune disease that is more severe in pediatric population than 
in adults. Biological therapy with anti-CD20 (rituximab) is an option in patient that does not respond to conventional therapy.

Methods The aim of this study is to determine the clinical and immunological response in 10 patients with cSLE that received treatment with rituximab in a third level hospital. 10 patients treated with Rituximab between November 2007 and October 2018 was included in a retrospective observational study. The response to treatment at 6 months and one year after the first infusion of Rituximab were assessed. All patients fulfilled four or more of the 1982 revised American College of Rheumatology criteria for the diagnosis of SLE $\quad<16$ years).

Results Ten cSLE patients treated with rituximab were included, all of them were female. The age at diagnosis of SLE was a mean of 15,61 years. The mean time duration of disease was 87,55 months $(5255 \mathrm{~m}) .8$ patients were caucasians. Rituximab was indicated in $60 \%$ of patients for class IV of lupus nephritis (LN), in $10 \%$ for class III LN, $10 \%$ for Class II LN, $10 \%$ for severe cutaneous lupus, and for severe hematological manifestations in $10 \%$ of cases (haemolytic anemia). In addition, $60 \%$ of the patients had mucocutaneous and articular manifestations. The disease activity of all patients was assessed using SELENASLEDAI index pre rituximab infusion, the mean was 17,31 (833). All patients had low level of complement and $90 \%$ increased anti-DNA. In 90\% of cases the Rituximab was used as a rescue treatment and in a single case as a first line.

$90 \%$ of patients with renal involvement were previously treated with CF iv and/or mycophenolate. In case of cutaneous involvement the previous treatment was methotrexate, azathioprine (AZA) and Dapsone and in case of hemolytic anemia the treatments was AZA.

The treatment protocol was 1 gram $\times 2 \times 6 \mathrm{~m}$ in $8 / 10$ patients, $375 \mathrm{mg} / \mathrm{m}^{2} \times 4$ in $1 / 10$ cases and $600 \mathrm{mg}$ monthly for $5 \mathrm{~m}$ in the case of HAI. Five patients received more than 1 cycle. After the administration of Rituximab, the SELENA-SLEDAI activity index was 5,1. At 6 months a complete response was obtained in the case of hematological and cutaneous manifestations, in 3 cases of LN (proteinuria $<0.5 \mathrm{~g} /$ day) and partial response was obtained in 2 cases. Data were not analysed in 2 patients (death and less than 6 months of the first dose of rituximab). Patients with partial response and lack of response achieved complete response at 12 months. 2/9 patients had side effects (Rituximab pneumonitis in 1 case and infections in 2 cases). Mortality was $10 \%$ (1/10 patients: infection and lupus activity, SLEDAI pre rituximab=33)

Conclusions In our study, although it consisted of few patients, it was objected that Rituximab therapy in patients with cSLE is effective, reduces lupus activity index, especially in cases of renal, cutaneous and hematologic involvement, that don't respond to conventional therapy. It may be considered in the future as an effective alternative treatment at first line treatment.

Funding Source(s): None

\section{CHARACTERIZATION OF CELL-BOUND COMPLEMENT ACTIVATION PRODUCTS ON SLE PBMCS USING MASS CYTOMETRY}

Rebecca E Schriefer, Gabriel R Arguelles, Lynne M Mitchell, Stephen T Oh, John P Atkinson, Dennis E Hourcade, Alfred H Kim*. Washington University School of Medicine

10.1136/lupus-2019-Ism. 147
Background Complement plays a central role in SLE. Its activation by immune complexes drives type II and III hypersensitivity reactions and classical pathway activation, leading to inflammatory responses in target tissues. Its activation generates an array of bioactive soluble and cellbound complement activation products (CB-CAPs). Data are lacking detailing the types and quantities of each CBCAP on SLE immune cells. We speculate that discerning the patterns and impacts of complement signatures on SLE B cells will provide insight into the role of complement activation in the pathogenesis of SLE. To characterize the array of CB-CAPs on SLE immune cells, we created and validated a mass cytometry (MC) panel suitable for detecting over $20 \mathrm{CB}-\mathrm{CAPs}$ and complement receptors, then examined PBMCs from paired flare and inactive samples from patients with SLE.

Methods Adults with ACR- or SLICC-classified SLE $(n=5)$ were enrolled and consented for PBMC collection at Washington University School of Medicine. Paired samples were obtained, one during a flare and one after resolution of the flare. Frozen ficoll gradient isolated buffy coat PBMCs were thawed counted, filtered, and treated with cisplatin to confirm viability. Prepared cells were stained with metal-conjugated antibodies to measure surface complement proteins and markers identifying immune cell subset. Stained cells were treated with an Iridium intercalator stain, recounted, washed in Milli-Q water to remove salts, resuspended with a solution of bead standards to facilitate data normalization, and subjected to single cell MC. Sample to sample consistency is optimized by simultaneously examining multiple cell preparations barcoded with palladium metals. Data analysis was performed with Cytobank. Distinct cell sub-populations were identified and organized in a hierarchal fashion.

Results We found the highest frequency of C4d deposition (indicative of classical pathway activation) on B cells compared to $\mathrm{T}$ cells and monocytes during SLE flares. Surprising, only B cells had C3d, C5 (common pathway), and Bb (alternative pathway) CAPs with none observed on $\mathrm{T}$ cells or monocytes suggesting that incomplete classical pathway activation occurred on these cells. We also found that different B cell subsets differentially activated complement during flares. Transitional B cells universally had C4d and C5 but surprisingly had little $\mathrm{C} 3 \mathrm{~d}$, despite the presence of $\mathrm{Bb}$. Mature B cells, particularly the mature anergic subset, and memory B cells had C4d and C3d but little C5. Plasma cells/ plasmablasts were devoid of any CB-CAPs. Finally, during disease inactivity, very low levels of all CB-CAPs were observed on all PBMCs.

Conclusions We found a high level of CB-CAP deposition in immune cells obtained from subjects with a SLE flare, which was absent during disease inactivity. The types of CB-CAPs on PBMCs were not uniform, and our data suggest that certain immune cells appear regulate complement activation while others do not. The reasons for this are unclear, but it potentially opens up a previously undescribed heterogeneity in SLE. These pilot data demonstrate the feasibility of the MC complement panel on human samples and the power of this approach in discovering novel mechanisms of complement activation and regulation.

Funding Source(s): AHK: NIH/NIAMS R21 AR069833, Doris Duke Charitable Foundation 